EUROPEAN JOURNAL OF PURE AND APPLIED MATHEMATICS

Vol. 15, No. 1, 2022, 82-99

ISSN 1307-5543 - ejpam.com

Published by New York Business Global

\title{
Application of a heuristic method to solve nonlinear oscillators with irrational forces
}

\author{
O. González-Gaxiola ${ }^{1, *}$, J. Ruiz de Chávez ${ }^{2}$ \\ ${ }^{1}$ Departamento de Matemáticas Aplicadas y Sistemas, Universidad Autónoma Metropolitana- \\ Cuajimalpa. Vasco de Quiroga 4871, Santa Fe, Cuajimalpa, 05300, Mexico City, Mexico \\ 2 Departamento de Matemáticas, Universidad Autónoma Metropolitana-Iztapalapa. \\ San Rafael Atlixco 186, Col. Vicentina, Iztapalapa, 09340, Mexico City, Mexico
}

\begin{abstract}
This paper applies another amplitude-frequency relationship as of late established by Yazdi and Tehrani (Alexandria Engineering Journal 54 99-103, 2015) to analyze periodical solutions of nonlinear oscillators; such oscillators are considered emphatically nonlinear on the grounds that they contain an irrational force term. Estimation strategy is straightforward yet additionally helpful and its formulation depends on combining the energy balance technique with an uncommon collocation point. At long last, we uncover the convenience and proficiency of the proposed technique resolving three instances of conservative nonlinear oscillators in which the maximum relative error acquired is $2.5 \%$. Exposed models show that the strategy has a high precision to solve mechanical issues of both small and huge estimations of the oscillation amplitude. Finally, the methodology used can be very useful for the study of nonlinear oscillators in basic undergraduate physics and mechanics courses.
\end{abstract}

2020 Mathematics Subject Classifications: 34A34, 97M10, 97M50

Key Words and Phrases: Nonlinear oscillators, Periodic solution, Approximate frequency, Conservative oscillator, Duffing-relativistic oscillator, Jacobi polynomials

\section{Introduction}

Most of engineering problems, especially some oscillation equations are nonlinear, and in most cases it is difficult to solve such equations, especially analytically. Recently, it is exceptionally valuable to have creative and effective numerical techniques to understand nonlinear differential equations, since huge numbers of the models for depicting phenomena in both applied mathematics and engineering end up being given through nonlinear equations. In ongoing literature we can locate that a few analytical and estimated strategies have been created to solve nonlinear oscillators throughout the most recent decades, for example: The harmonic balance method [1,2], which in practice is used to replace the

${ }^{*}$ Corresponding author.

DOI: https://doi.org/10.29020/nybg.ejpam.v15i1.4172

Email addresses: ogonzalez@cua.uam.mx (O. González-Gaxiola) jrch@xanum.uam.mx (J. Ruiz de Chávez) 
non-linear forces in the oscillating systems by specially-constructed linear functions. The He's variational approach [3] which is widely used to obtain an approximate analytical solutions for nonlinear problems. The energy balance technique $[4,5,6]$, which can be seen as a Ritz method and leads to a very rapid convergence of the solution, and can be easily extended to other nonlinear oscillations. The Hamiltonian approach $[7,8]$, which is very simple but strongly depends upon the chosen location point. The use of special functions $[9,10]$. The max-min approach $[11,12]$, which has millennia history. The Adomian decomposition technique [13], consisting of decomposing the solution into rapidly convergent series. The variational iteration method $[14,15,16]$, based on the concepts of force and momentum, and homotopy perturbation $[17,18]$, which is an effective technique and convenient one for both weakly and strongly nonlinear equations. An investigation that can be an exceptionally valuable tool, where a large numbers of these techniques can be found in detail to take care of nonlinear issues of oscillatory type can be seen in [19, 20].

As of late, In [21] Yazdi and Tehrani improved improved exactness of the energy balance through Jacobi collocation technique to moderate nonlinear oscillators. In addition, we can see techniques for solving oscillatory problems for teaching purposes in $[22,23]$. In this paper, we will uncover the new strategy proposed in [21] and we will likewise apply it to discover approximate solutions of certain nonlinear differential equations that model emphatically nonlinear oscillators with a nonsensical force and have made a comparison with the specific solution. The present method has the versatility and advantages of numerical methods for being applied directly to highly nonlinear problems and also have the elegance and other benefits of analytical techniques.

\section{Solution method}

The technique that we will uncover straightaway and which the perusers can discover in [21] with more prominent detail, ends up being the combination of the energy balance strategy with points that have an extremely extraordinary collocation point. It is worth mentioning that many of the modern techniques for solving oscillatory problems have been established by J. H. He. If the reader wishes to go deeper into the study, origin and fundamentals of collocation methods, energy balance methods, variational methods and Hamiltonian techniques we recommend references [24, 25, 26, 27, 28, 29, 30, 31].

\subsection{The Jacobi polynomials and its basic properties}

The Jacobi polynomials are generally used to tackle numerous issues in mathematics, physics, chemistry, biology, engineering and applied sciences. The Jacobi polynomials with general parameters $(\alpha, \beta>-1)$ are a sequence of polynomials $P_{n}^{(\alpha, \beta)}(x),(n=0,1,2, \ldots)$, every one of degree $n$. 
The Jacobi polynomials $P_{n}^{(\alpha, \beta)}(x)$ are given explicitly by

$$
P_{n}^{(\alpha, \beta)}(x)=\frac{1}{2^{n}} \sum_{k=0}^{n}\left(\begin{array}{c}
n+\alpha \\
n-k
\end{array}\right)\left(\begin{array}{c}
n+\beta \\
k
\end{array}\right)(x-1)^{k}(x+1)^{n-k},
$$

or, equivalently, by the well-known Rodrigues formula

$$
(x-1)^{\alpha}(x+1)^{\beta} P_{n}^{(\alpha, \beta)}(x)=\frac{(-1)^{n}}{2^{n} n !} \frac{d^{n}}{d x^{n}}\left[(1-x)^{n+\alpha}(1+x)^{n+\beta}\right], \quad x \in[-1,1] .
$$

Let $w^{(\alpha, \beta)}(x)=(x-1)^{\alpha}(x+1)^{\beta}$ be the Jacobi weight function. For $\alpha, \beta>-1$, the Jacobi polynomials satisfy the following orthogonality relation:

$$
\int_{-1}^{1} P_{n}^{(\alpha, \beta)}(x) P_{m}^{(\alpha, \beta)}(x) w^{(\alpha, \beta)}(x) d x=C_{n}^{\alpha, \beta} \delta_{n, m}
$$

where $\delta_{n, m}$ is the Kronecker function, and

$$
C_{n}^{\alpha, \beta}=\frac{2^{\alpha+\beta+1} \Gamma(n+\alpha+1) \Gamma(n+\beta+1)}{n !(2 n+\alpha+\beta+1) \Gamma(n+\alpha+\beta+1)} .
$$

The Jacobi polynomial is the solution the following second-order, homogeneous differential equation:

$$
\left(1-x^{2}\right) y^{\prime \prime}+[\beta-\alpha-x(\alpha+\beta+2)] y^{\prime}+n(n+\alpha+\beta+1) y=0 .
$$

The following recurrence relation generates the Jacobi polynomials [32]

$$
\begin{gathered}
P_{n+1}^{(\alpha, \beta)}(x)=\left(a_{n}^{(\alpha, \beta)} x-b_{n}^{(\alpha, \beta)}\right) P_{n}^{(\alpha, \beta)}(x)-c_{n}^{(\alpha, \beta)} P_{n-1}^{(\alpha, \beta)}(x), \quad n \geq 1 \\
P_{0}^{(\alpha, \beta)}(x)=1, \quad P_{1}^{(\alpha, \beta)}(x)=\frac{1}{2}((\alpha+\beta+2) x+\alpha-\beta),
\end{gathered}
$$

where

$$
\begin{gathered}
a_{n}^{(\alpha, \beta)}=\frac{(2 n+\alpha+\beta+1)(2 n+\alpha+\beta+2)}{2(n+1)(n+\alpha+\beta+1)}, \\
b_{n}^{(\alpha, \beta)}=\frac{\left(\beta^{2}-\alpha^{2}\right)(2 n+\alpha+\beta+1)}{2(n+1)(n+\alpha+\beta+1)(2 n+\alpha+\beta)}, \\
c_{n}^{(\alpha, \beta)}=\frac{(n+\alpha)(n+\beta)(2 n+\alpha+\beta+2)}{(n+1)(n+\alpha+\beta+1)(2 n+\alpha+\beta)} .
\end{gathered}
$$




\subsection{Basic idea of energy balance method with a special collocation point}

Consider a one-dimensional, free nonlinear oscillator (undamped and unforced) governed by

$$
\ddot{u}+f(u)=0,
$$

with the initial conditions

$$
u(0)=A, \quad \dot{u}(0)=0,
$$

where a dot denotes differentiation with respect to the time $t, u$ is the dimensionless displacement, $A$ will be a given constant, and the nonlinear restoring force $f(u)$ is an odd function of $u$, i.e. $f(-u)=-f(u)$ for $u \in[-A, A]$. Clearly $u=0$ is the equilibrium position. The system oscillates between the symmetric limits $-A$ and $A$. In the event that $f(u)$ is a nonlinear function, both period $T$ and frequency $\omega=2 \pi / T$ of the relating oscillation are reliant upon the amplitude of oscillation $A$. The connection between the frequency and amplitude is the main property of a nonlinear oscillator; see [33, 34, 35, 36, $37,38]$ and references therein.

The variational principle applied to Eq. (11) can be expressed as

$$
V(u)=\int_{0}^{t}\left\{-\frac{1}{2} \dot{u}^{2}+F(u)\right\} d \hat{t},
$$

in which, $F(u)=\int f(u) d u$. Hence, its Hamiltonian function becomes

$$
H=\frac{1}{2} \dot{u}^{2}+F(u)=F(A)
$$

or equivalently

$$
R(t)=\frac{1}{2} \dot{u}^{2}+F(u)-F(A)=0 .
$$

According to the initial conditions (12), the trial function for Eq. (11) is introduced to determine the angular frequency:

$$
u(t)=A \cos (\omega t) .
$$

Substituting (16) into (15), the corresponding residual turns out to be [39]:

$$
R(t)=\frac{1}{2} A^{2} \omega^{2} \sin ^{2}(\omega t)+F(A \cos (\omega t))-F(A) .
$$

According to the classical energy balance method [39, 40, 41], we have

$$
\omega_{E B M}(A)=\frac{\sqrt{2(F(A)-F(A \cos (\omega t)))}}{A \sin (\omega t)},
$$

with the collocation point in $\omega t=\frac{\pi}{4}$. 
As of late, the regular energy balance method has been changed by M. K. Yazdi and P. $\mathrm{H}$. Tehrani in [21], utilizing an extraordinary collocation point. As per the investigation done in [21], the approximate frequency as a function of A can be gotten as follows:

$$
\omega_{\text {app }}(A)=\frac{\sqrt{2\left(F(A)-F\left(A \cos \left(x_{0} \pi\right)\right)\right)}}{A \sin \left(x_{0} \pi\right)},
$$

where $x_{0}$ is the root of some one-degree Jacobi polynomial, this is, $P_{1}^{(\alpha, \beta)}\left(x_{0}\right)=0$. Both Jacobi polynomial parameters $(\alpha ; \beta>-1)$ can be utilized to acquire an optimal estimation of $x_{0}$. The precision relies extraordinarily on the collocation point.

From (19) we get the following approximate periodic solution for (11)

$$
u_{\text {app }}(t)=A \cos \left(\omega_{\text {app }} \cdot t\right) .
$$

The technique to find $x_{0}$ is an open problem, however we have chosen it in a heuristic way.

\section{Numerical examples}

In this section, we provide three examples to illustrate the use, ease and effectiveness of the proposed method. In each example we compare our results with the exact results found in the literature to show that the proposed method is accurate and efficient. It is not the objective of the present study to compare the method with other existing methods since we intend to expose this technique for didactic purposes only.

\section{Example 1.}

The governing non-dimensional nonlinear differential equation of motion for the relativistic oscillator is [42]:

$$
\ddot{u}+\frac{u}{\sqrt{1+u^{2}}}=0, \quad u(0)=A, \quad \dot{u}(0)=0,
$$

where $f(u)=\frac{u}{\sqrt{1+u^{2}}}$. Calculating we have

$$
F(u)=\int f(u) d u=\int \frac{u}{\sqrt{1+u^{2}}} d u=\sqrt{1+u^{2}},
$$

therefore, we obtain

$$
F(A)=\sqrt{1+A^{2}} \text { and } F\left(A \cos \left(\pi x_{0}\right)\right)=\sqrt{1+A^{2} \cos ^{2}\left(\pi x_{0}\right)},
$$

and the exact frequency is given by [41]:

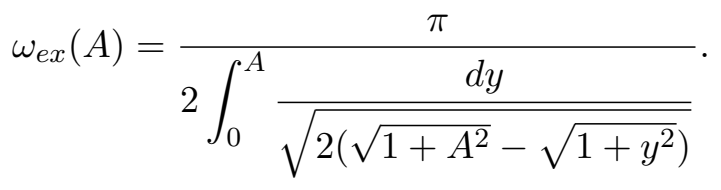

So as to apply the previously mentioned method for finding the approximate frequency and solution of the oscillator $(21)$, we propose to consider the Jacobi polynomial $P_{1}^{(864,1441)}(x)$, 
whose root is $x_{0}=0.250108$.

Choosing the collocation point $\omega t=0.250108 \pi$, the analytical approximation to periodic solution of the nonlinear oscillator is

$$
u(t)=A \cos \left(\omega_{\text {app }}(A) t\right),
$$

where

$$
\omega_{\text {app }}(A)=\frac{\sqrt{2 \sqrt{1+A^{2}}-2 \sqrt{1+A^{2} \cos ^{2}(0.250108 \pi)}}}{A \sin (0.250108 \pi)},
$$

which has a high accuracy, see Figs. 1 and 2.

Table 1 shows a comparison between the approximate frequencies $\omega_{\text {app }}(A)$ and the exact frequencies $\omega_{e x}(A)$ for different values of $A$, a decent solution is acquired.

\begin{tabular}{|c|c|c|c|}
\hline$A$ & $\omega_{\text {app }}(A)$ Eq. $(26)$ & $\omega_{\text {ex }}(A)$ Eq. $(24)$ & Relative Error $(\%)$ \\
\hline 0.01 & 0.999981 & 0.999981 & $0.00 \%$ \\
0.1 & 0.998135 & 0.998135 & $0.00 \%$ \\
0.5 & 0.958123 & 0.958338 & $0.02 \%$ \\
1 & 0.870586 & 0.872342 & $0.20 \%$ \\
5 & 0.477493 & 0.486634 & $1.87 \%$ \\
10 & 0.341110 & 0.349115 & $2.29 \%$ \\
50 & 0.153064 & 0.157029 & $2.52 \%$ \\
100 & 0.108244 & 0.111062 & $2.53 \%$ \\
1000 & 0.034231 & 0.035124 & $2.54 \%$ \\
10000 & 0.010824 & 0.011107 & $2.54 \%$ \\
100000 & 0.003423 & 0.003512 & $2.54 \%$ \\
\hline
\end{tabular}

Table 1: Comparison between frequencies $\omega_{\text {app }}(A)$ and $\omega_{e x}(A)$ for different values of $A$. 


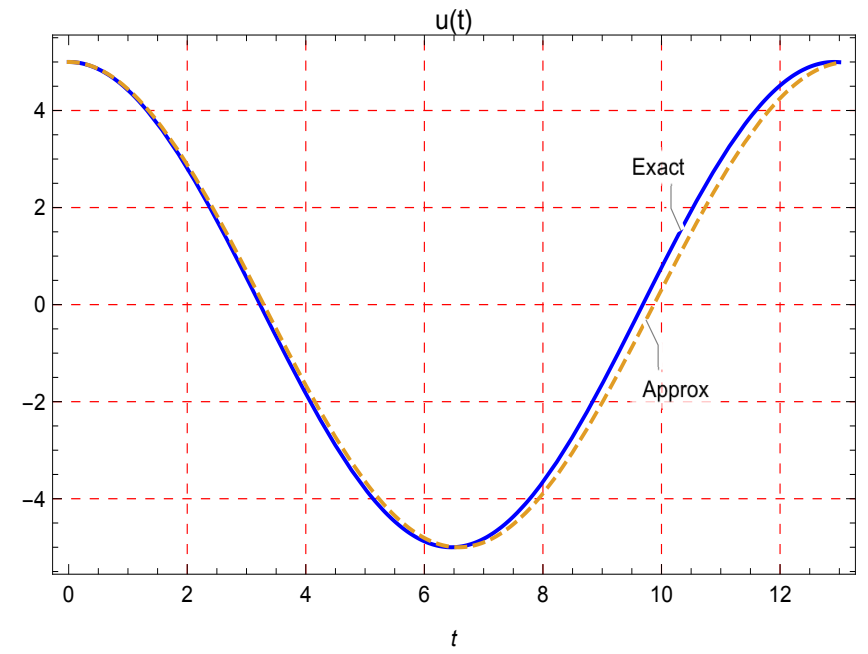

Figure 1: Example 1: comparison of the approximate periodic solution (dashed) with exact solution (blue) for $A=5$.

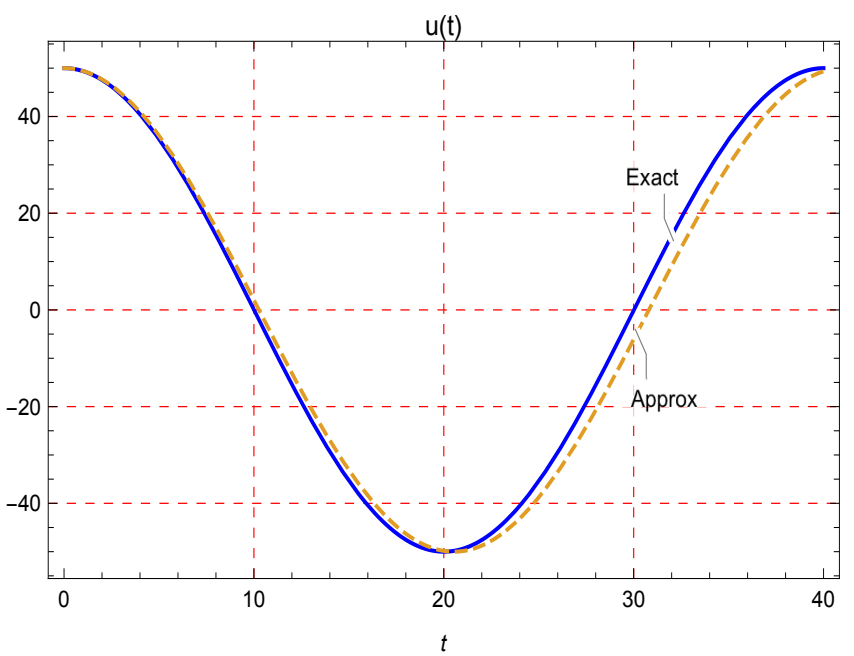

Figure 2: Example 1: comparison of the approximate periodic solution (dashed) with exact solution (blue) for for $A=50$.

We consider our approximation to be acceptable, since the best approximation to this problem had shown a maximum error of $1.6 \%$ for all the range of values of amplitude of oscillation $A$ [43].

\section{Example 2}

The motion equation for a mass attached to a stretched elastic wire is governed by the 
mathematical model with initial conditions given by $[1,8]$

$$
\ddot{u}+u-\frac{\gamma u}{\sqrt{1+u^{2}}}=0, \quad u(0)=A, \quad \dot{u}(0)=0, \quad 0<\gamma<1 .
$$

If $f(u)=u-\frac{\gamma u}{\sqrt{1+u^{2}}}$, calculating we have

$$
F(u)=\int f(u) d u=\frac{u^{2}}{2}-\gamma \sqrt{1+u^{2}} .
$$

Considering the above, we obtain

$$
\begin{aligned}
& F(A)=\frac{A^{2}}{2}-\gamma \sqrt{1+A^{2}} \\
& F\left(A \cos \left(\pi x_{0}\right)\right)=\frac{A^{2} \cos ^{2}\left(\pi x_{0}\right)}{2}-\gamma \sqrt{1+A^{2} \cos ^{2}\left(\pi x_{0}\right)} .
\end{aligned}
$$

The exact vibration frequency $\omega_{e x}$ can be derived by direct integration of governing Eq. (27) as follows [41, 44]:

$$
\omega_{e x}(A)=\pi\left(2 \int_{0}^{A} \frac{d y}{\sqrt{\left(A^{2}-y^{2}\right)-2 \gamma\left(\sqrt{1+A^{2}}-\sqrt{1+y^{2}}\right)}}\right)^{-1} .
$$

Case $\gamma=0.25$

For this situation, we propose to consider the Jacobi polynomial $P_{1}^{(856,1425)}(x)$ whose root is $x_{0}=0.24923$. By choosing the collocation point $\omega t=0.24923 \pi$, the approximation to periodic solution of the nonlinear oscillator is

$$
u(t)=A \cos \left(\omega_{\text {app }}(A) t\right),
$$

where

$$
\omega_{\text {app }}(A)=\frac{\sqrt{A^{2} \sin ^{2}(0.24923 \pi)+(0.5)\left[\sqrt{1+A^{2} \cos ^{2}(0.24923 \pi)}-\sqrt{1+A^{2}}\right]}}{A \sin (0.24923 \pi)} .
$$




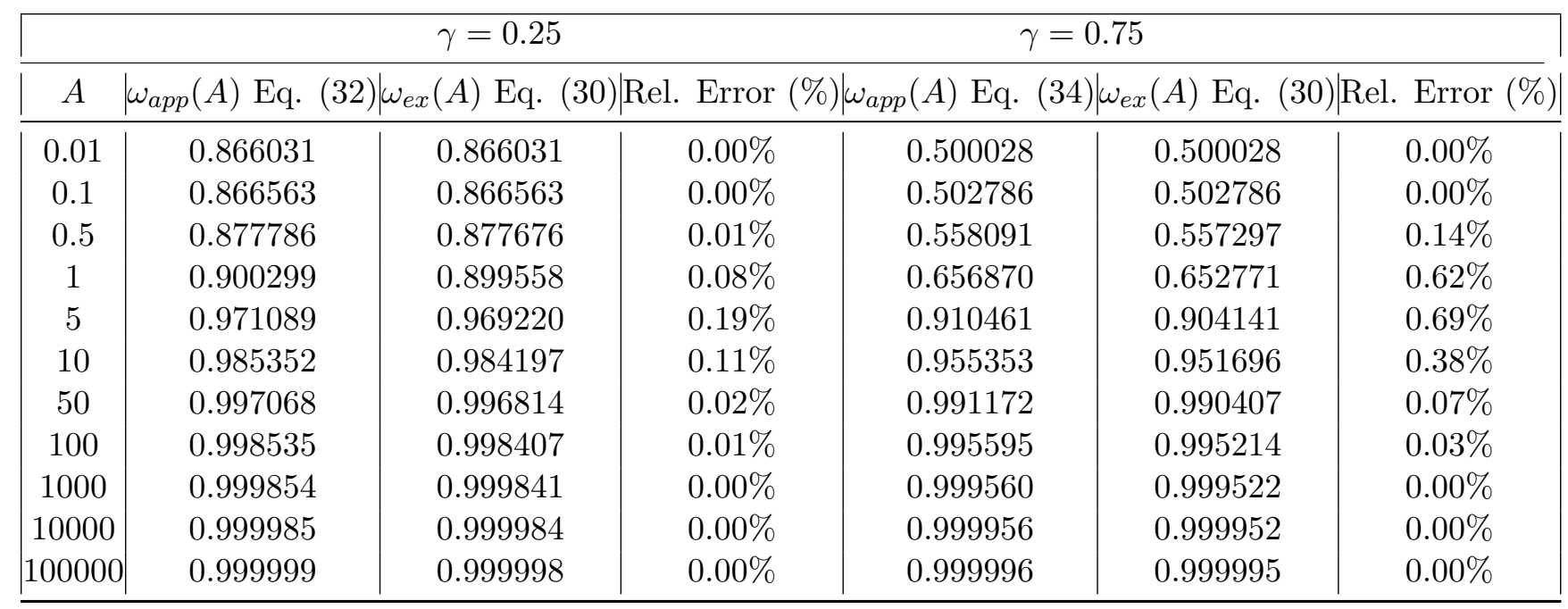

Table 2: Comparison between frequencies $\omega_{\text {app }}(A)$ and $\omega_{e x}(A)$ for different values of $A$.

Case $\gamma=0.75$

Here we propose to consider the Jacobi polynomial $P_{1}^{(457,763)}(x)$ whose root is $x_{0}=$ 0.250409. Besides we we choose the collocation point $\omega t=0.250409 \pi$, the approximation to periodic solution of the nonlinear oscillator is

$$
u(t)=A \cos \left(\omega_{a p p}(A) t\right),
$$

where

$$
\omega_{\text {app }}(A)=\frac{\left.\sqrt{A^{2} \sin ^{2}(0.250409 \pi)+(1.5)\left[\sqrt{1+A^{2} \cos ^{2}(0.250409 \pi)}-\sqrt{1+A^{2}}\right.}\right]}{A \sin (0.250409 \pi)} .
$$

In order to illustrate that our obtained result is a nice approximation to the exact solution, we compute numerical examples assuming $A=50$ and $\gamma=0.25,0.75$, see Fig. 3 and Fig. 4, respectively, and also Table 2 . 


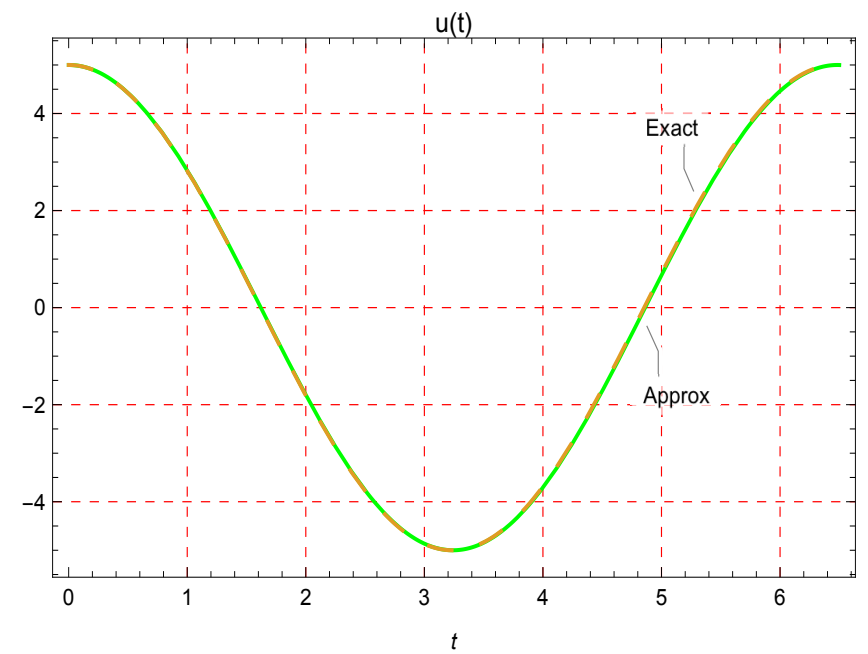

Figure 3: Example 2: comparison of the approximate periodic solution (dashed) with exact solution (green) for $A=50$ and $\gamma=0.25$.

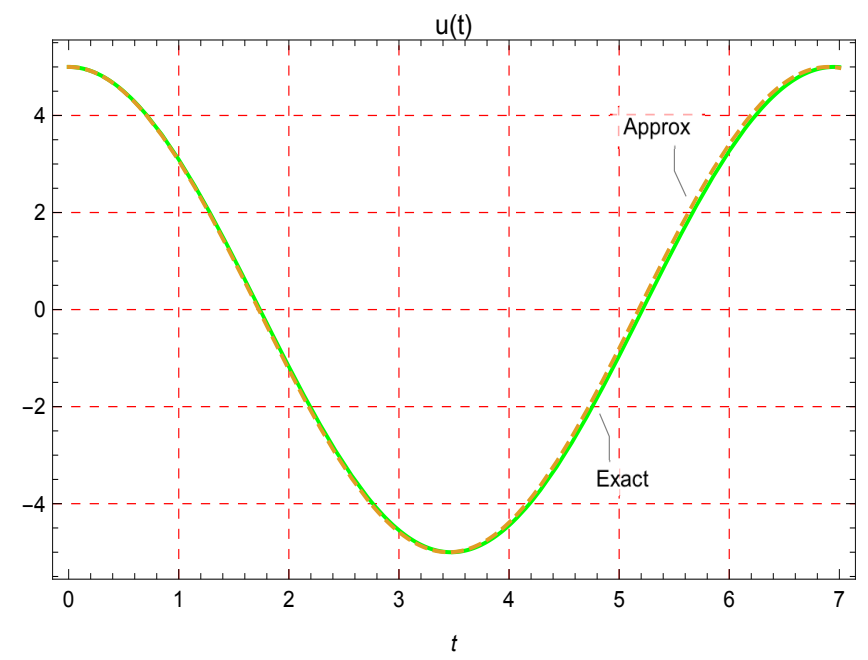

Figure 4: Example 2: comparison of the approximate periodic solution (dashed) with exact solution (green) for $A=50$ and $\gamma=0.75$.

We consider our approximation to be acceptable, since the best approximation to this problem had shown a maximum error of $0.8 \%$ for all the range of values of amplitude of oscillation $A[4]$.

Example 3 
Consider the Duffing-relativistic oscillator [45]:

$$
\ddot{u}+u+u^{3}-\frac{\lambda u}{\sqrt{1+u^{2}}}=0, \quad u(0)=A, \quad \dot{u}(0)=0,
$$

where $0<\lambda<1$ is a constant. Since $f(u)=u+u^{3}-\frac{\lambda u}{\sqrt{1+u^{2}}}$, we get

$$
F(u)=\int f(u) d u=\frac{u^{2}}{2}+\frac{u^{4}}{4}-\lambda \sqrt{1+u^{2}} .
$$

As a consequence, we have

$$
\begin{gathered}
F(A)=\frac{A^{2}}{2}+\frac{A^{4}}{4}-\lambda \sqrt{1+A^{2}}, \\
F\left(A \cos \left(\pi x_{0}\right)\right)=\frac{A^{2} \cos ^{2}\left(\pi x_{0}\right)}{2}+\frac{A^{4} \cos ^{4}\left(\pi x_{0}\right)}{4}-\lambda \sqrt{1+A^{2} \cos ^{2}\left(\pi x_{0}\right)} .
\end{gathered}
$$

We remark that Eq. (35) is a numerical model of a conservative system. The exact frequency $\omega_{e x}$ of this oscillator is given as [45]:

$$
\omega_{e x}(A)=\pi\left(2 \int_{0}^{A} \frac{d y}{\sqrt{\left(A^{2}-y^{2}\right)+\frac{1}{2}\left(A^{4}-y^{4}\right)-2 \lambda\left(\sqrt{A^{2}+1}-\sqrt{y^{2}+1}\right)}}\right)^{-1} .
$$

Case $\lambda=0.5$

For this value, we consider the Jacobi polynomial $P_{1}^{(891,1487)}(x)$ whose root is $x_{0}=0.25042$. Moreover, we take $\omega t=0.25042 \pi$ and consider the proposed approach in Eq. (19). Hence one can assume for the frequency-amplitude formulation

$$
\begin{aligned}
\omega_{\text {app }}(A)=\frac{1}{A \sin (0.25042 \pi)}( & A^{2} \sin ^{2}(0.25042 \pi)+\frac{A^{4}}{2}-\sqrt{A^{2}+1} \\
& \left.-\frac{A^{4}}{2} \cos ^{4}(0.25042 \pi)+\sqrt{1+A^{2} \cos ^{2}(0.25042 \pi)}\right)^{1 / 2}
\end{aligned}
$$

On the other hand, if we consider the approximation given by (20), we have obtain the following periodic solution of the equation (35)

$$
u_{\text {app }}(t)=A \cos \left(\omega_{\text {app }}(A) t\right)
$$

where $\omega_{\text {app }}(A)$ is given explicitly by the Eq. (40).

Case $\lambda=0.9$ 
Here, we consider the Jacobi polynomial $P_{1}^{(925,1549)}(x)$ whose root is $x_{0}=0.252019$.

Choosing the collocation point $\omega t=0.252019 \pi$, the analytical approximation to periodic solution of the nonlinear oscillator is

$$
u(t)=A \cos \left(\omega_{a p p}(A) t\right)
$$

where

$$
\begin{aligned}
\omega_{\text {app }}(A)=\frac{1}{A \sin (0.252019 \pi)}\left(A^{2} \sin ^{2}(0.252019 \pi)+\frac{A^{4}}{2}-\frac{A^{4}}{2} \cos ^{4}(0.25042 \pi)\right. \\
\left.+\frac{9}{5}\left[\sqrt{1+A^{2} \cos ^{2}(0.252019 \pi)}-\sqrt{A^{2}+1}\right]\right)^{1 / 2}
\end{aligned}
$$

Now, by taking the approximation given by Eq. (20), we have obtained the following periodic solution, for the case $\lambda=0.9$ of the Eq. (35)

$$
u_{\text {app }}(t)=A \cos \left(\omega_{\text {app }}(A) t\right)
$$

where $\omega_{\text {app }}(A)$ is explicitly given by the Eq. (43).

The numerical results are illustrated in the Figs. 5 and 6 . In Table 3 compares the approximate frequency with the exact one.

\begin{tabular}{|c|c|c|c|c|c|c|}
\hline \multicolumn{5}{|c|}{$\lambda=0.5$} \\
\hline$A$ & $\omega_{\text {app }}(A)$ Eq. $(40)$ & $\omega_{\text {ex }}(A)$ Eq. (39) & Rel. Error $(\%)$ & $\omega_{\text {app }}(A)$ Eq. $(43)$ & $\omega_{\text {ex }}(A)$ Eq. $(39) \mid$ Rel. Error $(\%)$ \\
\hline 0.01 & 0.707173 & 0.707173 & $0.00 \%$ & 0.316399 & 0.316400 & $0.00 \%$ \\
0.1 & 0.713692 & 0.713692 & $0.00 \%$ & 0.332881 & 0.332881 & $0.00 \%$ \\
0.5 & 0.853412 & 0.851625 & $0.20 \%$ & 0.600200 & 0.594202 & $1.00 \%$ \\
1 & 1.170609 & 1.160531 & $0.86 \%$ & 1.031538 & 1.014874 & $1.64 \%$ \\
5 & 4.429387 & 4.342080 & $2.01 \%$ & 4.411920 & 4.329715 & $1.89 \%$ \\
10 & 8.710673 & 8.529475 & $2.12 \%$ & 8.693554 & 8.526183 & $1.96 \%$ \\
50 & 43.29364 & 42.37283 & $2.17 \%$ & 43.22095 & 42.37269 & $2.00 \%$ \\
100 & 86.57018 & 84.72744 & $2.17 \%$ & 86.42497 & 84.72740 & $2.00 \%$ \\
1000 & 865.6450 & 847.2137 & $2.17 \%$ & 864.1931 & 847.2137 & $2.00 \%$ \\
10000 & 8656.444 & 8472.131 & $2.17 \%$ & 8641.925 & 8472.131 & $2.00 \%$ \\
100000 & 86564.44 & 84721.31 & $2.17 \%$ & 86419.25 & 84721.31 & $2.00 \%$ \\
\hline
\end{tabular}

Table 3: Comparison between frequencies $\omega_{\text {app }}(A)$ and $\omega_{e x}(A)$ for different values of $A$. 


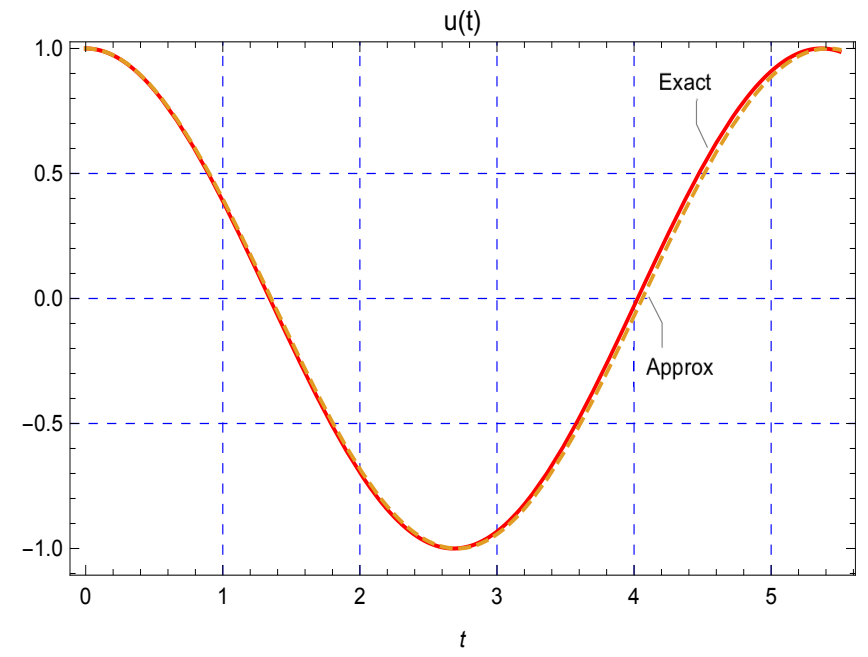

Figure 5: Comparison of the approximate periodic solution (dashed) with exact solution (red) for $A=1$ and $\lambda=0.5$.

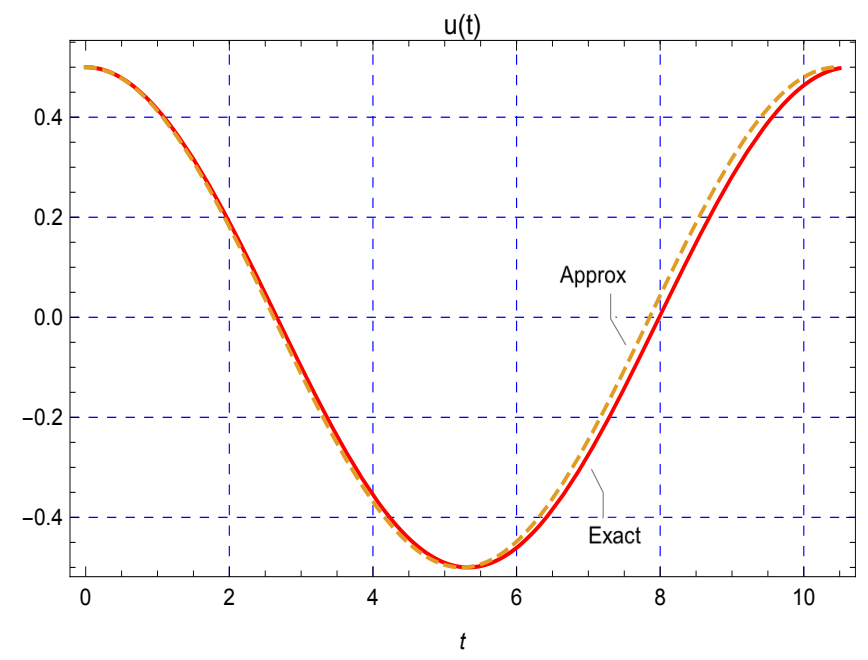

Figure 6: Comparison of the approximate periodic solution (dashed) with exact solution (red) for $A=0.5$ and $\lambda=0.9$.

We consider our approximation to be acceptable, since the best approximation to this problem had shown a maximum error of $2.2 \%$ for all the range of values of amplitude of oscillation $A[39]$. 


\section{Conclusions}

Another amplitude-frequency relationship as of late established in [21] is proved being a ground-breaking numerical tool for use in the quest for periodic solutions of nonlinear oscillators. It is basic, clear and effective. Also, the approximate analytical solutions are suitable for both small and large estimations of oscillation amplitude and parameter. It is worth mentioning that the problem of finding a way to find the point $x_{0}$ as a function of the parameters of the Jacobi polynomial remains open.

At last, three physical models have been resolved to outline the effectiveness and applicability of the proposed solution approach and the outcomes in Tables 1, 2 and 3 and Figs. $1,2,3,4,5$ and 6 uncover that this strategy can be considered as a practical option for traditional techniques which can resolve exceptionally nonlinear oscillatory frameworks. All numerical work and illustrations were performed with the Mathematica software package.

The present method can be used to introduce the study of nonlinear vibrations and oscillators in undergraduate sciences and engineering courses due to its simplicity and accuracy for a wide range of problems. It can also add pedagogical value to undergrads from various branches of natural sciences and engineering, in particular mechanical engineering students by introducing the Jacobi polynomials in a pleasant manner. This is because the present methodology applies these polynomials in a simple scheme that does not make it seem of great mathematical difficulty.

\section{References}

[1] Ronald E Mickens. Oscilations in planar dynamic systems. World Scientific, Cop, 1996.

[2] Ali Hasan Nayfeh. Problems in perturbation. Wiley, Repr, 1993.

[3] M.K. Yazdi. "Approximate solutions to nonlinear oscillations via an improved He's variational approach". In: Karbala International Journal of Modern Science 2 (2016), pp. 289-297. DOI: 10.1016/j.kijoms.2016.11.003.

[4] Akuro Big-Alabo and Collins Onyinyechukwu Ogbodo. "Dynamic analysis of crank mechanism with complex trigonometric nonlinearity: a comparative study of approximate analytical methods". In: SN Applied Sciences 1 (2019). DOI: 10.1007/s42452019-0673-3. (Visited on 11/19/2021).

[5] Y. Khan and A. Mirzabeigy. "Improved accuracy of He's energy balance method for analysis of conservative nonlinear oscillator". In: Neural Computing and Applications 25 (2014), pp. 889-895. DOI: $10.1007 /$ s00521-014-1576-2. (Visited on $11 / 19 / 2021)$.

[6] A. Yildirim et al. "Analysis of nonlinear oscillations of a punctual charge in the electric field of a charged ring via a Hamiltonian approach and the energy balance method". In: Computers 83 Mathematics with Applications 62 (2011), pp. 486-490. DOI: $10.1016 / \mathrm{j}$. camwa.2011.05.029. (Visited on 11/19/2021). 
[7] Ji-Huan He. "Hamiltonian approach to nonlinear oscillators". In: Physics Letters A 374 (2010), pp. 2312-2314. DOI: 10.1016/j.physleta.2010.03.064. (Visited on $11 / 19 / 2021)$.

[8] Ahmet Yildirim, Z. Saadatnia, and H. Askari. "Application of the Hamiltonian approach to nonlinear oscillators with rational and irrational elastic terms". In: Mathematical and Computer Modelling 54 (2011), pp. 697-703. DOI: $10.1016 / \mathrm{j} . \mathrm{mcm}$. 2011.03.012. (Visited on 11/19/2021).

[9] Alex Elías-Zúñiga. "Exact solution of the cubic-quintic Duffing oscillator". In: Applied Mathematical Modelling 37 (2013), pp. 2574-2579. DOI: 10.1016/j .apm. 2012. 04.005. (Visited on 11/19/2021).

[10] Alex Elías-Zúñiga. "Solution of the damped cubic-quintic Duffing oscillator by using Jacobi elliptic functions". In: Applied Mathematics and Computation 246 (2014), pp. 474-481. DOI: $10.1016 / \mathrm{j}$. amc.2014.07.110.

[11] J.H. He. "Max-Min Approach to Nonlinear Oscillators". In: International Journal of Nonlinear Sciences and Numerical Simulation 9 (2008). DOI: 10.1515/ijnsns. 2008.9.2.207. (Visited on 11/12/2020).

[12] M. Kalami Yazdi et al. "Dynamic Analysis of Vibrating Systems with Nonlinearities". In: Communications in Theoretical Physics 57 (2012), pp. 183-187. DOI: 10. 1088/0253-6102/57/2/03. (Visited on 01/03/2020).

[13] O. González-Gaxiola, J. A. Santiago, and J. Ruiz de Chávez. "Solution for the Nonlinear Relativistic Harmonic Oscillator via Laplace-Adomian Decomposition Method". In: International Journal of Applied and Computational Mathematics 3 (2016), pp. 2627-2638. DOI: 10.1007/s40819-016-0267-3. (Visited on 11/19/2021).

[14] Ji-Huan He. "Variational approach for nonlinear oscillators". In: Chaos, Solitons \& Fractals 34 (2007), pp. 1430-1439. DOI: 10.1016/j.chaos.2006.10.026. (Visited on $11 / 19 / 2021)$.

[15] A. Mirzabeigy, M. Kalami-Yazdi, and A. Yildirim. "Analytical approximations for a conservative nonlinear singular oscillator in plasma physics". In: Journal of the Egyptian Mathematical Society 20 (2012), pp. 163-166. DOI: $10.1016 /$ j . joems . 2012.05.001. (Visited on 01/03/2020).

[16] M. Rafei et al. "The variational iteration method for nonlinear oscillators with discontinuities". In: Journal of Sound and Vibration 305 (2007), pp. 614-620. DOI: 10.1016/j.jsv.2007.04.020. (Visited on 11/19/2021).

[17] Ji-Huan He. "Homotopy perturbation method for solving boundary value problems". In: Physics Letters A 350 (2006), pp. 87-88. DOI: 10.1016/j.physleta.2005.10. 005. (Visited on 11/19/2021).

[18] Ji-Huan He. "Homotopy Perturbation Method for Bifurcation of Nonlinear Problems". In: International Journal of Nonlinear Sciences and Numerical Simulation 6 (2005). DOI: 10.1515/ijnsns.2005.6.2.207. (Visited on 11/19/2021). 
[19] L. Cveticanin, M. Kalami-Yazdi, and H. Askari. "Analytical approximations to the solutions for a generalized oscillator with strong nonlinear terms". In: Journal of Engineering Mathematics 77 (2012), pp. 211-223. DOI: 10.1007 /s10665-0129542-4. (Visited on 01/03/2020).

[20] Ji-Huan He. "Some asymptotic methods for strongly nonlinear equations". In: International Journal of Modern Physics B 20 (2006), pp. 1141-1199. DOI: 10.1142/ s0217979206033796. (Visited on 11/19/2021).

[21] M.K. Yazdi and P.H. Tehrani. "The energy balance to nonlinear oscillations via Jacobi collocation method". In: Alexandria Engineering Journal 54 (2015), pp. 99103. DOI: $10.1016 /$ j . aej .2015.03.016. (Visited on 11/19/2021).

[22] Akuro Big-Alabo. "A simple cubication method for approximate solution of nonlinear Hamiltonian oscillators". In: International Journal of Mechanical Engineering Education 48 (2019), pp. 241-254. DOI: 10.1177/0306419018822489. (Visited on $11 / 19 / 2021)$.

[23] Akuro Big-Alabo. "Approximate periodic solution for the large-amplitude oscillations of a simple pendulum". In: International Journal of Mechanical Engineering Education 48 (2019), pp. 335-350. DOI: 10.1177/0306419019842298. (Visited on 07/08/2021).

[24] Ji-Huan He et al. "Hamiltonian-based frequency-amplitude formulation for nonlinear oscillators". In: Facta Universitatis, Series: Mechanical Engineering 19 (2021), p. 199. DOI: 10.22190/fume201205002h. (Visited on 11/19/2021).

[25] Ji-Huan He. "The simpler, the better: Analytical methods for nonlinear oscillators and fractional oscillators". In: Journal of Low Frequency Noise, Vibration and Active Control 38 (2019), pp. 1252-1260. DOI: 10.1177/1461348419844145. (Visited on $11 / 19 / 2021)$.

[26] Ji-Huan He. "The simplest approach to nonlinear oscillators". In: Results in Physics (2019), p. 102546. DOI: 10.1016/j.rinp.2019.102546.

[27] Yue Wu and Ji-Huan He. "Homotopy perturbation method for nonlinear oscillators with coordinate-dependent mass". In: Results in Physics 10 (2018), pp. 270-271. DOI: $10.1016 /$ j.rinp. 2018.06.015.

[28] Ji-Huan He. "Amplitude-Frequency Relationship for Conservative Nonlinear Oscillators with Odd Nonlinearities". In: International Journal of Applied and Computational Mathematics 3 (2016), pp. 1557-1560. DOI: 10.1007/s40819-016-0160-0. (Visited on 11/19/2021).

[29] Ji-Huan He. "An elementary introduction to the homotopy perturbation method". In: Computers 83 Mathematics with Applications 57 (2009), pp. 410-412. DOI: 10 . 1016/j . camwa. 2008.06.003. (Visited on 11/19/2021).

[30] Ji-Huan He, Yusry O. El-Dib, and Amal A. Mady. "Homotopy Perturbation Method for the Fractal Toda Oscillator". In: Fractal and Fractional 5 (2021), p. 93. DOI: 10.3390/fractalfract5030093. (Visited on 11/19/2021). 
[31] Ji-Huan He. "Comment on 'He's frequency formulation for nonlinear oscillators". In: European Journal of Physics 29 (2008), pp. L19-L22. DOI: 10 . 1088/01430807/29/4/102. (Visited on 11/19/2021).

[32] Ben-Yu Guo, Jie Shen, and Li-Lian Wang. "Generalized Jacobi polynomials/functions and their applications". In: Applied Numerical Mathematics 59 (2009), pp. 10111028. DOI: $10.1016 / j$. apnum. 2008.04.003. (Visited on 11/19/2021).

[33] O. González-Gaxiola. "Periodic Solution for Strongly Nonlinear Oscillators by He's New Amplitude-Frequency Relationship". In: International Journal of Applied and Computational Mathematics 3 (2017), pp. 1249-1259. DOI: 10.1007/s40819-0170414-5. (Visited on 11/19/2021).

[34] Xu-Chu Cai and Jun-Fang Liu. "Application of the modified frequency formulation to a nonlinear oscillator". In: Computers $\& 3$ Mathematics with Applications 61 (2011), pp. 2237-2240. DOI: 10.1016/j . camwa.2010.09.025. (Visited on 11/19/2021).

[35] S. S. Ganji et al. "Periodic Solution for Strongly Nonlinear Vibration Systems by He's Energy Balance Method". In: Acta Applicandae Mathematicae 106 (2008), pp. 7992. DOI: $10.1007 / \mathrm{s} 10440-008-9283-6$. (Visited on 11/19/2021).

[36] Y. Khan et al. "Dynamic Analysis of Generalized Conservative Nonlinear Oscillators Via Frequency Amplitude Formulation". In: Arabian Journal for Science and Engineering 38 (2011), pp. 175-179. DOI: 10.1007/s13369-011-0035-y. (Visited on $11 / 19 / 2021)$.

[37] M. K. Yazdi. "An accurate relationship between frequency and amplitude to nonlinear oscillations". In: Journal of Taibah University for Science 12 (2018), pp. 532535. DOI: 10.1080/16583655.2018.1498290. (Visited on 11/19/2021).

[38] M Kalami Yazdi and P Hosseini Tehrani. "Frequency analysis of nonlinear oscillations via the global error minimization". In: Nonlinear Engineering 5 (2016), pp. 8792. DOI: $10.1515 / \mathrm{nleng}-2015-0036$. (Visited on 01/03/2020).

[39] Ji-Huan He. "Preliminary report on the energy balance for nonlinear oscillations". In: Mech. Res. Commu. 29 (2002), pp. 107-111. (Visited on 2002).

[40] Ronald E Mickens. Truly nonlinear oscillations : harmonic balance, parameter expansions, iteration, and averaging methods. World Scientific, Cop, 2010.

[41] Davood Younesian et al. "Frequency analysis of strongly nonlinear generalized Duffing oscillators using He's frequency-amplitude formulation and He's energy balance method". In: Computers $\& 3$ Mathematics with Applications 59 (2010), pp. 3222-3228. DOI: $10.1016 /$ j . camwa. 2010.03.013. (Visited on 10/17/2021).

[42] R.E. Mickens. "Periodic solutions of the relativistic harmonic oscillators". In: Journal of Sound and Vibration 212 (1998), pp. 905-908. DOI: 10.1006/jsvi.1997.1453. (Visited on 11/19/2021). 
[43] A. Beléndez et al. "Application of a modified He's homotopy perturbation method to obtain higher-order approximations of an force nonlinear oscillator". In: Physics Letters A 371 (2007), pp. 421-426. DOI: 10.1016/j . physleta. 2007 . 06 . 042. (Visited on 11/19/2021).

[44] W.P. Sun, B.S. Wu, and C.W. Lim. "Approximate analytical solutions for oscillation of a mass attached to a stretched elastic wire". In: Journal of Sound and Vibration 300 (2007), pp. 1042-1047. DOI: 10.1016/j.jsv.2006.08.025. (Visited on $11 / 19 / 2021)$.

[45] D. Younesian et al. "Analytical approximate solutions for the generalized nonlinear oscillator". In: Applicable Analysis 91 (2012), pp. 965-977. DOI: 10.1080/00036811. 2011.559464. (Visited on 11/19/2021). 